\title{
New Information on the Occurrence of the $O(6)$ Symmetry in Nuclei
}

\author{
N. Pietralla ${ }^{1, a}$, T. Möller ${ }^{1}$, C.J. Lister ${ }^{2,3}$, E.A. McCutchan ${ }^{2,4}$, G. Rainovski ${ }^{5}$, C. Bauer ${ }^{1}$, M.P. Carpenter ${ }^{2}$, \\ R.V.F. Janssens ${ }^{2}$, D. Seweryniak ${ }^{2}$, and S. Zhu² \\ ${ }^{1}$ Institut für Kernphysik, Technische Universiät Darmstadt, Schlossgartenstr. 9, 64289 Darmstadt, Germany \\ ${ }^{2}$ Physics Division, Argonne National Laboratory, Argonne, IL 60439, USA \\ ${ }^{3}$ Department of Physics and Applied Physics, University of Massachusetts Lowell, Lowell, MA 01854, USA \\ ${ }^{4}$ National Nuclear Data Center, Brookhaven National Laboratory, Upton, NY 11973, USA \\ ${ }^{5}$ Faculty of Physics, St. Kliment Ohridski University of Sofia, 1164 Sofia, Bulgaria
}

\begin{abstract}
New $\gamma \gamma$-coincidence relations and E2 decay transition rates in the isotopes ${ }^{194,196} \mathrm{Pt}$ have been deduced from $\gamma$-ray spectroscopy experiments using the Gammasphere spectrometer in projectile-Coulomb excitation reactions of beams of ${ }^{194,196} \mathrm{Pt}$ ions provided by the ATLAS accelerator facility. The results give access to observables that are crucial for a classification of excited quadrupole-collective states in terms of quantum numbers associated with the analytically solvable $\mathrm{O}(6)$ dynamical symmetry of the interacting boson model. The data on ${ }^{196} \mathrm{Pt}$ corroborate the qualitative pattern of excitation energies and $E 2$ transition rates expected from the $\mathrm{O}(6)$ solution but the excitation energies significantly deviate from it on a quantitative level.
\end{abstract}

\section{Introduction}

The interacting boson model (IBM) is an algebraic model capable of the description of collective valence shell excitations of atomic nuclei [1]. Its Hamiltonian problem can be solved analytically in particular non-trivial situations called dynamical symmetries. The symmetries are associated with the occurrence of quantum numbers and corresponding selection rules for decay transitions between nuclear states, that are useful for their classification. In this way the IBM provides benchmarks for nuclear structure and helps to simplify their analysis and interpretation.

The $s d$-IBM-1 [1] deals with low-energy quadrupolecollective nuclear structure. Besides others, it supports an $\mathrm{O}(6)$ dynamical symmetry whose eigenstates can be classified in terms of quantum numbers $N, \sigma, \tau$, and $L$ associated with eigenvalues of Casimir operators of the Liegroups $\mathrm{U}(6), \mathrm{O}(6), \mathrm{O}(5)$, and $\mathrm{O}(3)$, respectively. Excited states occur at excitation energies

$$
E_{x}(N ; \sigma, \tau, L)=a[N(N+4]-\sigma(\sigma+4)]+b \tau(\tau+3)+c L(L+1)
$$

with the reduction rules $\sigma=N, N-2, \ldots(1$ or 0$), \tau=0$, $1, \ldots, \sigma$, and various values for the angular momentum quantum number $L \leq 2 \tau$ as a function of $\tau$. The wave functions corresponding to these eigenvalues are independent of the values of $a, b$, and $c$ in Eq. (1) which, therefore, can phenomenologically be adjusted for a best match with data. For positive constants $a$ and $b$, as it results from an attractive quadrupole-quadrupole interaction, and a nonnegative value $c$ for the centrifugal term, it is easy to see

\footnotetext{
ae-mail: pietralla@ikp.tu-darmstadt.de
}

that the ground state and the lowest-energy states have an $\mathrm{O}(6)$ quantum number $\sigma=N$ and $\mathrm{O}(5)$ symmetry. The $\mathrm{O}(6)$ symmetry then predicts a repetition of structural patterns with $\mathrm{O}(5)$ symmetry at higher excitation energies and $\mathrm{O}(6)$ quantum number $\sigma=N-2, N-4$, etc. Verification of this repetition of peculiar structural features can serve as a stringent test of the occurrence of the $\mathrm{O}(6)$ dynamical symmetry. It requires the observation of excited states with $\mathrm{O}(6)$ quantum number $\sigma=N-2$ etc. and measurement of their properties.

The first claim for an observation of a sequence of energy levels with $\mathrm{O}(6)$ quantum numbers $\sigma=N-2$ was made by Casten, Cizewski and others [2] from the analysis of $\gamma$-decay branching ratios from excited states of ${ }^{196} \mathrm{Pt}$. They indicate their compliance with selection rules for $E 2$ transitions that are forbidden unless the $\mathrm{O}(5)$ quantum numbers of the initial and final states differ by $\Delta \tau= \pm 1$ and the $\mathrm{O}(6)$ quantum numbers stay unchanged, $\Delta \sigma=0$. Since then, ${ }^{196} \mathrm{Pt}$ is considered the best manifestation of $\mathrm{O}(6)$ symmetry in nuclei with a $\sigma=N-2$ assignment to the $J^{\pi}=0^{+}, 2^{+}, 2^{+}$states at excitation energies of 1403 , 1604 , and $1847 \mathrm{keV}$, respectively. While a small E2 decay strength [3] and a considerable decay rate via electron conversion [4] have been measured on the $0^{+}$"band head" of the assigned $\sigma=N-2$ structure, it has neither been shown that the excited sequence is quadrupole-collective, indeed, nor that it exhibits $\mathrm{O}(5)$ symmetry, too, as it ought to be the case for $\mathrm{O}(6)$ dynamical symmetry to hold. Furthermore, it has not been tested up to now if the absolute values of collective $E 2$ transitions between excited states with $\sigma=N-2$ agree quantitatively with the $\mathrm{O}(6)$ symmetry, simply because these data are not measured. 
Recently, some of us have investigated absolute E2 transition rates of excited states of some Xenon and Barium isotopes in the mass range $A \approx 130$. Also these nuclei have previously been argued to exhibit $\mathrm{O}(6)$ dynamical symmetry, based on the observation of $\tau$-selection rules related to $\mathrm{O}(5)$ symmetry [5]. Our recent data on ${ }^{124,126} \mathrm{Xe}[6,7]$, however, demonstrate the existence of (at least moderately) collective $E 2$ transitions between states with different $\mathrm{O}(6)$ quantum numbers $\sigma$ which is at variance with the selection rule for $E 2$ transitions with respect to $\sigma$. A quantitative quantum number fluctuation analysis indicates a severe breaking of $\mathrm{O}(6)$ symmetry, at least for the isotopes ${ }^{124,126} \mathrm{Xe}$. These data were obtained from a sequence of ultra-high count-rate $\gamma$-ray spectroscopy measurements in projectile-Coulomb excitation reactions of Xenon beams provided by the ATLAS accelerator facility at Argonne National Laboratory. The $\gamma$-rays were measured with the Gammasphere detector array running in single- $\gamma$ detection mode. Due to the demonstrated sensitivity of the method to quantities that are decisive on the $\mathrm{O}(6)$ character of excited states, we have recently performed such measurements on the isotopes ${ }^{194,196} \mathrm{Pt}$, in order to obtain more information on the properties of states with $\mathrm{O}(6)$ quantum number assignments $\sigma=N-2$.

\section{Experiments}

Projectile-Coulomb excitation experiment have been performed at Argonne National Laboratory. Beams of ${ }^{194,196} \mathrm{Pt}$ ions have been accelerated by the ATLAS accelerator and collided onto a thin carbon target. The beam energies have been adjusted to $85 \%$ of the Coulomb barrier. Deexcitation $\gamma$-rays have been observed with the Gammasphere spectrometer operated in singles mode. Figure 1 shows a part of the $\gamma$-ray spectrum from ${ }^{194} \mathrm{Pt}$.

We stress the enormous count rate of several hundreds of millions of counts on the dominant peak of the ground

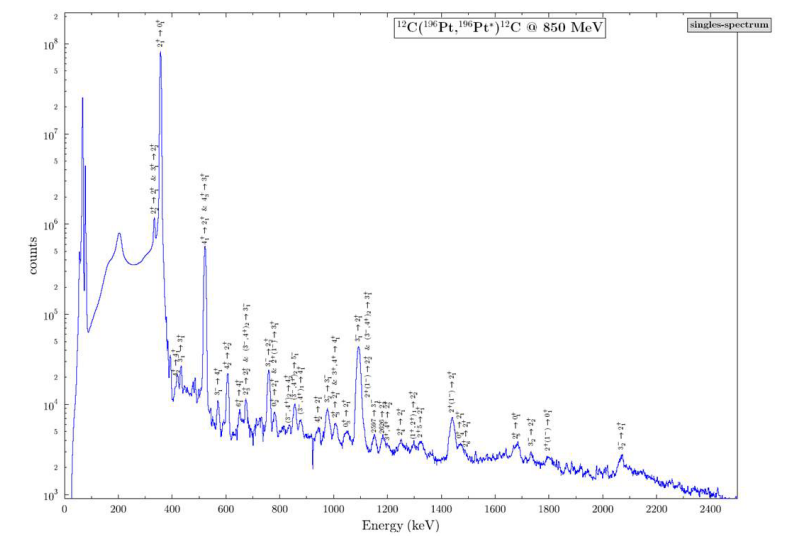

Figure 1. Pulse height spectrum of $\gamma$-rays detected with the Gammasphere array of $\mathrm{HPGe}$-detectors in the ${ }^{\text {nat }} \mathrm{C}\left({ }^{194} \mathrm{Pt},{ }^{194} \mathrm{Pt}^{*}\right)$ projectile-Coulomb excitation reaction at $850 \mathrm{MeV}$ beam energy. The data acquisition was triggered by single $\gamma$ events. Natural $\gamma$ ray background was subtracted using the time-correlation of the beam pulses to the radio-frequency signals from the ATLAS linear ion accelerator. Taken from Ref. [8]. state decay line of the $2_{1}^{+}$state and the sensitivity to higherenergy $\gamma$-ray lines with four to five orders of magnitude smaller intensities. About $2 \%$ of the events have a $\gamma$-ray multiplicity of 2 or higher that have been sorted into $\gamma \gamma$ coincidence matrices. The primary observables are the $\gamma$-ray intensities from which angular distributions, decay branching ratios, and yields of Coulomb excitation of the individual energy levels relative to the yield of the $2_{1}^{+}$state have been determined.

The relative reaction yields can be converted into absolute Coulomb excitation cross sections via the known $B(E 2)$ excitation strengths of the $2_{1}^{+}$states of the ${ }^{194,196} \mathrm{Pt}$ nuclei. The Coulomb excitation cross sections have been determined with the help of a computer code for the calculation of Coulomb excitation cross sections from electromagnetic matrix elements that were given as inputs and were varied systematically for a best match with the experimental data. The computer programs GOSIA and CLX have been used. The analysis was simplified by our choice of the light target material of carbon. Multi-step excitations that complicate the analysis were strongly suppressed. The influence of unknown relative phases of matrix elements for multi-step excitations has been included in the systematical uncertainties of the deduced electromagnetic matrix elements.

\section{Results}

In ${ }^{194} \mathrm{Pt}$ we have observed two close-lying $0^{+}$states above the first excited $0^{+}$with $\mathrm{O}(5)$ quantum number $\tau=3$. One of them decays by 12 and 15 W.u.(E2) to the low-lying $2_{1,2}^{+}$ states with $\mathrm{O}(6)$ quantum number $\sigma=N$. The observation of these moderately collective $E 2$ transition rates rule out a pure $\sigma=N-2$ assignment to this state. Similarly to the situation in ${ }^{124,126} \mathrm{Xe},{ }^{194} \mathrm{Pt}$ must be considered as a nucleus for which $\mathrm{O}(6)$ symmetry is considerably broken.

In ${ }^{196} \mathrm{Pt}$ we have observed only one $0^{+}$state above the first excited $0^{+}$with $\mathrm{O}(5)$ quantum number $\tau=3$. It is the previously observed $0^{+}$state at $1402 \mathrm{keV}$ excitation energy that has been assigned the $\mathrm{O}(6)$ quantum number $\sigma=N-2$ and $\mathrm{O}(5)$ quantum number $\tau=0$. It decays non-collectively with a strength of $3(2)$ W.u.(E2) to the $2_{1}^{+}$ state in qualitative agreement with its $\mathrm{O}(6)$ quantum number assignment $\sigma=N-2$. This result of our measurement is supported by the data obtained from lifetime measurements with the fast timing method performed at the ILL and presented by Professor Dr. Jan Jolie from the University of Cologne at this conference.

Our data provide evidence for a strongly collective $E 2$ transition with a strength of $2.0(7)(10) \times 10^{2}$ W.u. $(E 2)$ from the $2^{+}$state at excitation energy $1604 \mathrm{keV}$ to the $0_{3}^{+}$state at $1402 \mathrm{keV}$. The numbers in parentheses denote statistical and systematical uncertainties, respectively. Clearly, this $E 2$ transition is collective. It represents first direct evidence for the claim that the $0^{+}$state at $1402 \mathrm{keV}$ indeed supports a quadrupole-collective structure. All other decay branches to lower-lying states with $\mathrm{O}(6)$ quantum number assignments $\sigma=N$ have much smaller strengths of less than 4 W.u.(E2), each. This decay pattern is qualitatively in agreement with the expectations for the $\Delta \sigma=0$ 


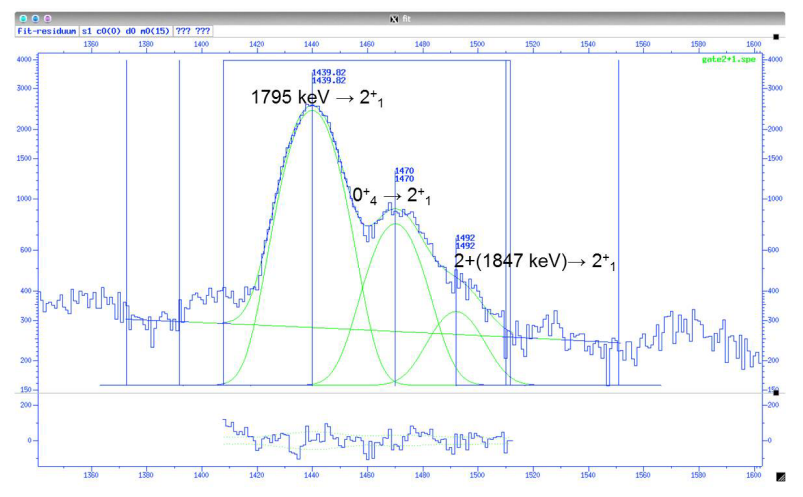

Figure 2. Relevant part of the $\gamma \gamma$-coincidences gated with the ground state decay of the $2_{1}^{+}$state. The peak to the right originates in the decay of the $2^{+}$state at $1847 \mathrm{keV}$, assigned $\sigma=N-2$ and $\tau=2$, to the $2_{1}^{+}$state at $356 \mathrm{keV}$.

and $\Delta \tau= \pm 1$ selection rules for $E 2$ transitions from a $2^{+}$ with $\mathrm{O}(6)$ quantum number $\sigma=N-2$ and $\mathrm{O}(5)$ quantum number $\tau=1$. However, the experimental E2 transition strengths have very large uncertainties. Therefore, we cannot draw any quantitative conclusions on the extent of possible $\mathrm{O}(6)$ symmetry breaking from the observed $E 2$ transition rates. The energy difference between this $2^{+}$state and the $0^{+}$at $1402 \mathrm{keV}$ with the $\sigma=N-2$ assignment amounts to $202 \mathrm{keV}$. This value should be equal to the excitation energy of the $2_{1}^{+}$state at $356 \mathrm{keV}$ if the $\mathrm{O}(6) \mathrm{dy}-$ namical symmetry, and hence Eq. (1), would be expected to hold exactly. This is obviously not the case for the moments of inertia.

Our data are also sensitive to the $2^{+}$state at $1847 \mathrm{keV}$ excitation energy which has previously been assigned as the $2^{+}$state with $\mathrm{O}(6)$ quantum number $\sigma=N-2$ and $\mathrm{O}(5)$ quantum number $\tau=2$. According to the selection rules it is expected to decay with a collective $E 2$ transition to the state with quantum number assignments $\sigma=N-2$ and $\tau=1$ made to the $2^{+}$state at $1604 \mathrm{keV}$. Figure 2 shows a relevant part of the $\gamma \gamma$-coincidences gated with the ground state decay of the $2_{1}^{+}$state. Observation of the $2_{1847 \mathrm{keV}}^{+} \rightarrow 2_{1}^{+}$transition together with the branching ratios known for the decay of the level at $1847 \mathrm{keV}$ enables us to determine the cross section for its Coulomb excitation and a lower limit for the decay rate of the $2_{1847 \mathrm{keV}}^{+} \rightarrow 2_{1604 \mathrm{keV}}^{+}$ transition. Assuming pure $E 2$ character we obtain a limit of $B\left(E 2 ; 2_{1847 \mathrm{keV}}^{+} \rightarrow 2_{1604 \mathrm{keV}}^{+}\right)>30(14)(13)$ W.u.(E2) again indicating a collective character. Other decay transition strengths from this state to lower-lying states with assignments $\sigma=N$ and $\tau<3$ are smaller than this by factors of more than 100. This finding is in qualitative agreement with the $\mathrm{O}(6)$ dynamical symmetry, too.

\section{Conclusions}

The nucleus ${ }^{194} \mathrm{Pt}$ features two close-lying $0^{+}$states near 1.5 MeV. One of them decays with a moderately collective $E 2$ transition to the $2_{1}^{+}$state. This indicates considerable breaking of $\mathrm{O}(6)$ dynamical symmetry, very similar to the situation which has previously been found for the structure of ${ }^{124,126} \mathrm{Xe}$. The level scheme of ${ }^{196} \mathrm{Pt}$ contains, instead, a sequence of three excited states whose decay transition strengths are in qualitative agreement with the assignment of quantum numbers $\sigma=N-2$ and $\tau=0,1$, and 2. The new data provide first evidence for their $E 2 \mathrm{col}-$ lectivity. This, together with the apparent $\mathrm{O}(5)$ symmetry of the ground state structure, demonstrates the repetition of $\mathrm{O}(5)$ structures predicted for an $\mathrm{O}(6)$ dynamical symmetry.

\section{Acknowledgments}

N.P. thanks Peter von Brentano, Rick Casten, Jan Jolie, and Ami Leviatan for discussions. G.R. gratefully acknowledges support from the Alexander von Humboldt foundation. This work was supported by the German research Council, DFG, under grant Nos. Pi393/3-3 and SFB 643, by the Helmholtz International Center for FAIR funded by the State of Hesse under the LOEWE program, by the German Federal Ministry for Education and Research, BMBF, under grant No. 05P12RDCIB, and by the U.S. Department of Energy.

\section{References}

[1] F. Iachello and A. Arima, The Interacting Boson Model (Cambridge University Press, Cambridge, 1987).

[2] J. Cizewski et al., Phys. Rev. Lett. 40, 167 (1978).

[3] H. G. Börner, J. Jolie, S. Robinson, R. F. Casten, and J. Cizewski, Phys. Rev. C 42, R2271 (1990).

[4] W.R. Kane, R.F. Casten, D.D. Warner, K. Schreckenbach, H.R. Faust, and S. Blakeway, Phys. Lett. B117, 15 (1982).

[5] R. F. Casten and P. von Brentano, Phys. Lett. B152, 22 (1985).

[6] G. Rainovski, N. Pietralla, T. Ahn, L. Coquard, C. Lister, R. Janssens, M. Carpenter, S. Zhu, L. Bettermann, J. Jolie, et al., Phys. Lett. B683, 11 (2010).

[7] L. Coquard, G. Rainovski, N. Pietralla, T. Ahn, L. Bettermann, M. P. Carpenter, R. V. F. Janssens, J. Leske, C. J. Lister, O. Möller, et al., Phys. Rev. C 83, 044318 (2011).

[8] Thomas Möller, Aspects of nuclear collectivity studied in projectile Coulomb excitation experiments (Dissertation, TU Darmstadt, 2014). 
